\title{
CAMERO-VENOUS SHUNT FOR SECONDARY GLAUCOMA FOLLOWING ORBITAL VENOUS OBSTRUCTION*†
}

\author{
BY
}

\author{
I. SRI-SKANDA-RAJAH-SIVAYOHAM \\ Eye Hospital, Colombo, Ceylon
}

THE patency of the orbital venous system is a sine qua non for the successful drainage of aqueous humour, so that a constant level of ocular tension may be maintained. The fact that the orbital veins are devoid of valves would cause any rise in pressure in the orbital veins to be reflected simultaneously in a rise in intra-ocular pressure, almost in a linear relationship.

On the assumption that the orbital venous pressure remains unchanged in the vast majority of cases of glaucoma simplex, various fistulizing procedures have been advocated and performed, in order to harness the episcleral venous channels (and thereby the orbital venous system) for drainage of the aqueous. Thus, in operations such as corneo-scleral trephining, anterior sclerectomy, thermal sclerotomy, iridencleisis, and nylon filament trabeculotomy, the rationale is to establish a by-pass communication between the anterior chamber and the episcleral venous system.

There is an analogy between the aqueous dynamics in cases of orbital venous obstruction and the cerebrospinal fluid dynamics in hydrocephalus; and in the latter it is possible to shunt the cerebrospinal fluid by establishing a communication between its pathways and the vascular system. This approach was considered relevant in the case under review.

The treatment of hydrocephalus by ventriculo-venous anastomosis was first suggested by H. T. Gartner (1895) at a Science Convention in Lübeck, but it remained a theory until Erwin Payr (1908) attempted the first ventriculo-venous anastomosis. He employed a segment of the saphenous vein to establish a communication between the lateral ventricle and the longitudinal sinus. Payr later modified his techniques to anastomose the lateral ventricle with the internal jugular vein, with excellent results.

Nowadays we have at our disposal excellent plastic materials which, by virtue of their rigidity and tensile strength, overcome a great many of the drawbacks of the collapsible venous segment utilized in the era of Payr.

In this publication an entirely new technique of camero-venous shunt is described, whereby a communication was established between the anterior chamber and the ipsilateral superficial temporal vein - the indication being a secondary glaucoma following an orbital venous occlusion.

\section{Case Report}

A 55-year-old man presented with proptosis of the right eye of 18 months' duration. The proptosis was gradually progressive and for the last 4 months the eye had been painful. There was also diplopia in all positions of gaze corroborated by gross limitation of movement. $X$-ray examination

* Received for publication October 24, 1967.
$\dagger$ Address for reprints: $185 / 19$ Havelock Rd., Colombo 5, Ceylon. 
revealed a shadow in the region of the superior orbital fissure and a diagnosis of meningioma of the sphenoidal ridge was made.

Surgery.-In August, 1965, a meningioma was removed piecemeal by the neurosurgeon at the General Hospital, Colombo. The patient was followed up both at the neurosurgical department and at the Eye Hospital, Colombo.

Progress.-Approximately 9 months after the decompression he presented at my clinic at the Eye Hospital with severe pain in the right eye, marked congestion of the bulbar and palpebral conjunctiva, and pronounced chemosis. The vision in this eye was reduced to perception of light. In spite of severe corneal oedema, it was possible (after instilling hypertonic glycerin) to discern neovascularization on the surface of the iris. Tonometry revealed an intra-ocular pressure of $65 \mathrm{~mm}$. Hg (Schiötz).

Treatment.-The aetiological bearing of the orbital venous thrombosis on the rise in intra-ocular pressure having been recognized, it was decided to establish a shunt between the angular vein and the anterior chamber. The angular vein was chosen because it is the one closest to the orbit. The silicone tube selected had an internal diameter of $1.6 \mathrm{~mm}$. and an external diameter of $2.8 \mathrm{~mm}$.

The patient was given 4 days of acetazolamide and pilocarpine therapy before surgery was undertaken. General anaesthesia was employed. Pre-operatively the tension had fallen to $45 \mathrm{~mm}$. Hg Schiötz but not below this.

The angular vein was explored but, as its calibre was too small to accommodate the silicone tube, it was abandoned. Instead, the ipsilateral superficial temporal vein was identified and exposed, and held for purposes of identification with a stay suture. This exposure was completed right down to the point where the vein entered the substance of the parotid gland.

The lower eyelid was retracted downwards and laterally and the inferior conjunctival fornix incised, the edges being undermined in order to expose the inferior oblique insertion. The lower conjunctival flap was undermined so as to reach the subcutaneous plane overlying the orbital rim, and thence backwards and outwards, aided by an intervening guide-incision in the skin parallel to the upper margin of the zygomatic arch.

The upper surface of the inferior oblique belly was freed from the globe by dissection. A limbally-hinged anterior flap sclerotomy was formed in the 7 o'clock meridian. The silicone tube was then inserted through the conjunctival incision subcutaneously and past the guide-incision to emerge from the pre-auricular incision. The other end of the tube was then made to pass above the inferior oblique belly so that it might lie in a plane between the globe and the muscle. Two horizontal scleral mattress sutures were passed and tied over the silicone tube, thus assuring safe anchorage.

The end of the silicone tube was finally anchored by a silk suture attaching it to the overlying flap of the sclerotomy. This suture incorporated about a third of the wall of the tube. Thus about $2 \mathrm{~mm}$. of the free end of the tube was allowed to enter the anterior chamber. The flap sclerotomy was sutured back into place, so that no leakage of aqueous occurred. Attention was next paid to the other end of the tube, which was negotiated into the lumen of the superficial temporal vein through a transverse nick in its wall, including about 40 per cent. of its circumference. There was an almost concurrent reflux of blood into the anterior chamber, but this disappeared on ligating the vein above the site of entry. A ligature was then used to make the flow uni-directional towards the heart. The assumption that this flow of aqueous from a cavity of higher pressure to one of lower pressure takes place was proved correct on follow-up examination.

Result.-At the first dressing it was observed that the corneal oedema had subsided and the end of the tube in the anterior chamber remained well anchored. On the 5th day a faint gossamer-like coagulum was visible in the vicinity of the free end of the tube. The conjunctival sutures were then removed, and the skin sutures were removed on the 7th day. Healing was by first intention. Slit-lamn examination on the 7th day revealed regression of the neovascularization of the iris. 
There was a faint aqueous flare and a couple of posterior synechiae were present. These were overcome by mydriatic and steroid drops. The shunt was functioning efficiently and applanation tonometry readings of $12-14 \mathrm{~mm} . \mathrm{Hg}$ were found at subsequent visits.

The patient remained pain-free and his visual acuity improved to 6/18. He had a somewhat pale optic disc dating from the time of the pressure atrophy induced by the sphenoidal ridge meningioma.

There was a tendency for the posterior synechiae to re-establish themselves, but this was prevented by subconjunctival injections of hydrocortisone and mydricaine.

About 10 months after the operation the patient returned with a vitreous haemorrhage which cleared satisfactorily in four days, but there was never a hyphaema. It is now 14 months since the operation and the shunt continues to function, the visual acuity remains good and applanation tonometry reveals that the intra-ocular pressure is well-controlled.

Limitations.-This operation may not be practicable for all types of glaucoma, but may well be of value in glaucoma due to orbital vein thrombosis-a relatively rare condition.

\section{Summary}

An entirely new and successful operative procedure is described, which is designed to reduce intra-ocular pressure after orbital venous occlusion, instead of the time-honoured procedure of cyclodiathermy based on the principle of reducing secretion. The rationale is the same as that for ventriculo-venous anastomosis in cases of congenital hydrocephalus. 